\title{
Directed Evolution of Bicyclic Peptides for Therapeutic Application
}

\author{
Philippe Diderich and Christian Heinis*
}

\begin{abstract}
Many naturally occurring cyclic peptides or derivatives thereof are used as therapeutics such as the human hormones vasopressin and oxytocin or the antibiotics vancomycin and daptomycin. The success of cyclic peptide therapeutics is based on their ability to bind with high affinity, their good target selectivity and their low toxicity. As nature provides cyclic peptides to only a small number of disease targets, strategies have been developed to generate cyclic peptide ligands with tailored specificity de novo. Our laboratory is specialized on the directed evolution of bicyclic peptide ligands by phage display. In this article, we review our recent work to in vitro evolve bicyclic peptide antagonists, the binding and pharmacokinetic properties of bicyclic peptides, as well as efforts to generate bicyclic peptides for therapeutic application.
\end{abstract}

Keywords: Cyclic peptides · Directed evolution · Peptides · Phage display · Therapeutics

\section{Application of Cyclic Peptides as Therapeutics}

More than 20 cyclic peptides are clinically approved for the treatment of infectious diseases, cancer, cardiovascular diseases and other maladies. ${ }^{[1]}$ Examples of cyclic peptide drugs are the antibiotics daptomycin, vancomycin and polymyxin $\mathrm{b}$, the hormones and hormone analogues oxytocin, vasopressin and octreotide, and the immunosuppressive drug cyclosporine. Three cyclic peptides are among the 200 bestselling drugs, namely cyclosporine, daptomycin and octreotide.[2] The annual sales of cyclosporine exceed 1 billion dollar and the latter two drugs are each generating revenues of more than half a billion dollar per year.

Several properties make cyclic peptides attractive for therapeutic application. Firstly, they can bind with high affinity and selectivity to protein targets. In contrast to linear analogues, cyclic peptides are less flexible and the entropic penalty upon tar-

${ }^{*}$ Correspondence: Prof. Dr. C. Heinis Institute of Chemical Sciences and Engineering Ecole Polytechnique Fédérale de Lausanne $\mathrm{CH}-1015$ Lausanne

E-mail: christian.heinis@epfl.ch get binding is smaller. Cyclization also reduces the number of possible conformers and thus lowers the chance for off-target binding. Many peptide drugs bind with affinities in the low nanomolar or picomolar range and thus show therapeutic activities at low drug concentration. Secondly, most cyclic peptide drugs show no toxicity, even if applied at concentrations higher than the therapeutic dose. This can be attributed to their high selectivity as well as to the fact that degradation products are usually not toxic. Thirdly, due to the limited conformational flexibility, many cyclic peptides are relatively resistant to proteases although the stability can vary strongly from peptide to peptide.

All cyclic peptide drugs are naturally occurring peptides or derivatives thereof.[1] For many important disease targets, peptide ligands could not be identified in nature and peptide drugs were thus not developed. The number of peptide therapeutics would be much higher if peptide ligands with good binding properties were available to a larger range of therapeutic targets. An efficient way of generating peptide ligands with tailored specificity is by in vitro evolution as discussed in the following section.

\section{In vitro Evolution of Peptide Ligands}

Cyclic peptides binding to targets of interest can be developed efficiently by screening large combinatorial libraries using in vitro display techniques. ${ }^{[3]}$ The first such technique developed was phage display and works as follows:[4] Libraries of polypeptides are fused to a phage coat protein to become displayed on the surface of a bacteriophage. The encoding DNA is enclosed in the interior of the viral particle and thus physically connected to the polypeptide. A pool of billions of phage, all displaying different polypeptides, is subjected to affinity selection with an immobilized target. The DNA of captured phage is sequenced to identify the amino acid sequences of polypeptides binding to the target. Phage display was first applied to evolve linear peptides and antibodies. While phage-selected antibodies bound with high affinity and could be developed into therapeutics, ${ }^{[5]}$ isolated linear peptides bound only weakly and peptide phage display was mostly applied for antibody epitope mapping. ${ }^{[6]}$ In some phage selections with linear peptide libraries, pairs of cysteines were found in the peptide sequences, suggesting that the peptides were cyclized through disulfide bridges. ${ }^{[7]}$ The cyclic peptides showed significantly better binding affinities than the linear ones and they displayed also higher stability. Subsequently, phage libraries of random peptides flanked by two cysteines were cloned to evolve cyclic peptide ligands and selections with several targets yielded ligands with dissociation constants in the low nanomolar range. ${ }^{[8,9]}$ Since the early 1990s, cyclic peptides were isolated against more than hundred protein targets. ${ }^{[10,11]}$

Another in vitro display technique frequently applied for the directed evolution of cyclic peptides is mRNA display. ${ }^{[12]}$ In this technique, polypeptides are covalently linked to their encoding mRNA during translation in cell-free systems. Recently developed innovative tools and strategies have enabled the incorporation of nonnatural amino acids into mRNA-encoded peptides. ${ }^{[13,14]}$ This has led to the directed 
evolution of cyclic peptides containing non-natural amino acids. ${ }^{[15-18]}$

The binding affinities of most in vitro evolved cyclic peptides are in the micromolar or high nanomolar range and thus too weak for therapeutic application. ${ }^{[10]}$ From the few in vitro evolved cyclic peptides showing decent binding strengths, a first one was approved in 2012 for clinical use but its distribution was soon after halted due to adverse effects observed in some patients and related safety concerns. ${ }^{[19,20]}$ This molecule, with the generic name peginesatide (formerly named hematide), is an erythropoietin mimetic and based on a cyclic peptide isolated in phage selections against erythropoietin receptor. The peptide was dimerized and pegylated to enhance its pharmacologic activity.

\section{In vitro Evolution of Bicyclic Peptides}

Bicyclic peptides contain two connected macrocyclic rings that can both engage in binding (Fig. 1A). The two peptide rings can act much like complementarity determining regions (CDRs) of antibodies. The bicyclic peptides thus may be seen as small antibody mimetics (Fig. 1B). Compared to monocyclic analogues, the bicyclic structures are less flexible. The lower conformational freedom reduces the entropic penalty upon binding to a target as well as renders the peptide more resistant to proteases.

Bicyclic peptides with desired binding specificity can be evolved in vitro with a phage display-based approach developed by Winter and Heinis (Fig. 2). ${ }^{[21]}$ In brief, random peptides containing cysteine residues at both ends and one in the middle are displayed on filamentous phage and cyclized by reaction with 1,3,5-tris(bromomethyl)benzene (TBMB). ${ }^{[21,22]}$ The bicyclic peptide library is panned against immobilized protein targets and enriched peptides are chemically synthesized for testing their binding. In a proof of principle study, a library containing more than 4 billion different bicyclic peptides, each having six random amino acids per peptide ring (6x6 library), was subjected to 2-3 rounds of selection against the serine proteases plasma kallikrein and cathepsin $\mathrm{G}$. The best bicyclic peptides inhibited the proteases with $K_{\mathrm{i}} \mathrm{s}$ of 2.9 and $100 \mathrm{nM}$, respectively. ${ }^{[21]}$

We are applying the bicyclic peptide phage selection approach routinely in our laboratory to i) generate antagonists of proteins for potential therapeutic application, ii) develop antagonists for target validation and iii) to obtain reagents for use as research tools. A bicyclic peptide isolated against urokinase-type plasminogen

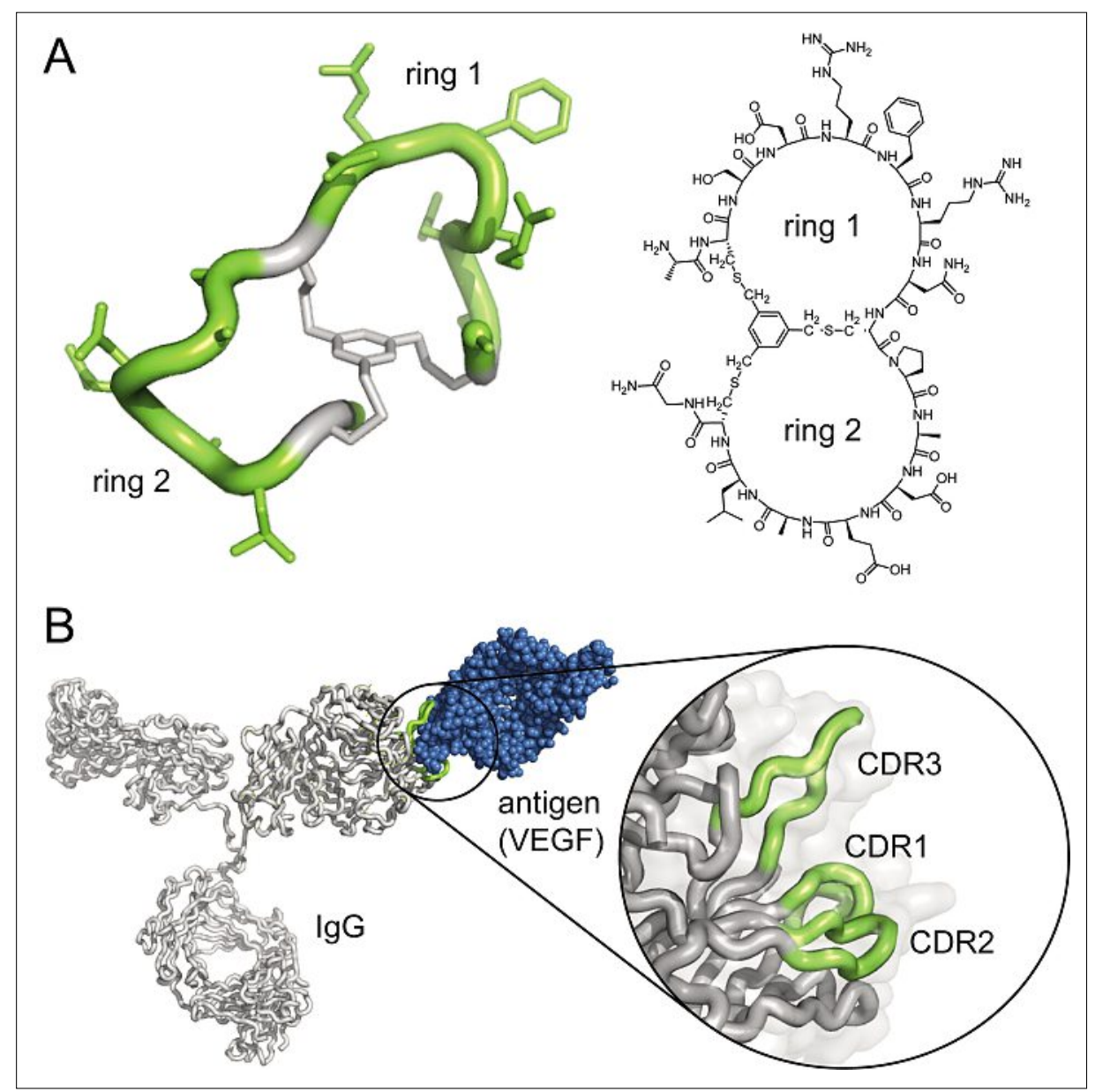

Fig. 1. Structure of a bicyclic peptide and its comparison with an antibody. (A) Structural model and chemical structure of the bicyclic peptide PK15. The peptide macrocycle was isolated by phage display and inhibits human plasma kallikrein with a $K_{\mathrm{i}}$ of $2.9 \mathrm{nM}$. (B) Structure of an antibody binding to VEGF. The complementarity determining regions (CDRs) of the variable heavy domain highlighted in green interact with VEGF. Bicyclic peptides may be seen as miniaturized antibodies with the two peptide rings mimicking the CDRs.

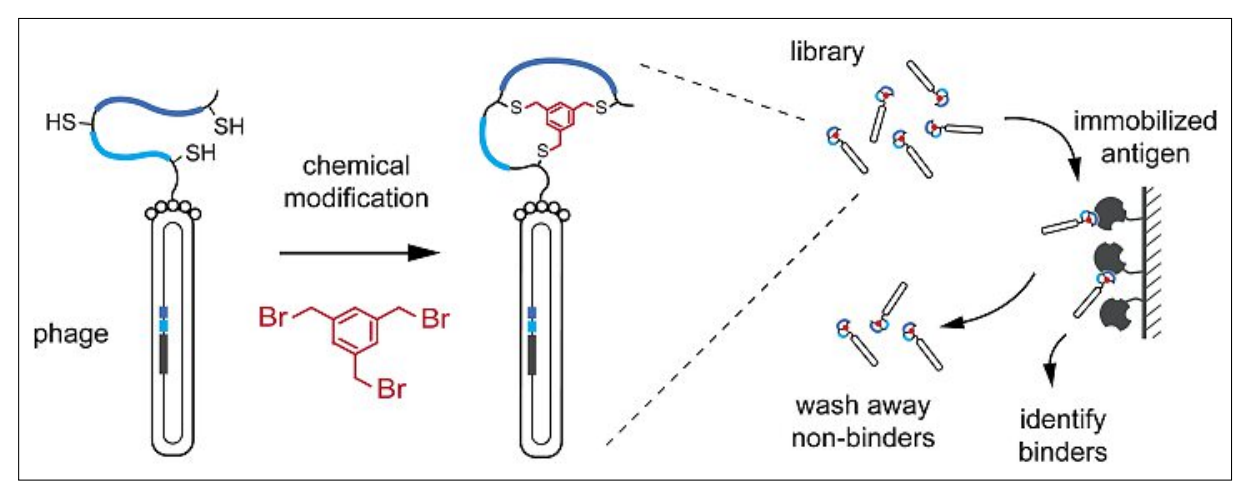

Fig. 2. Strategy for the phage selection of bicyclic peptides. Linear peptides containing two regions of random amino acids (in light and dark blue) spaced by three cysteines are displayed on filamentous phage and chemically cyclized by reaction with 1,3,5-tris(bromomethyl)benzene (in red). Billions of virions all displaying different bicyclic peptides are subjected to iterative rounds of affinity selection and amplification. The DNA of enriched phage is sequenced, the peptides synthesized chemically and their binding to the protein target measured.

activator (UPA) could be co-crystallized with the target, offering an insight into its binding mode (collaboration with Prof. Giuseppe Zanotti, University Padua; Fig. 3). ${ }^{[23]}$ Both peptide rings of the bicyclic peptide engaged in binding and formed with the target an interaction surface of $>900 \AA^{2}$. The numerous non-covalent inter- actions explained the good binding affinity $\left(K_{\mathrm{i}}=53 \mathrm{nM}\right)$ and selectivity (>1000-fold weaker inhibition of para- and orthologous proteases). Representation of the bicyclic peptide as space-fill model in the co-crystal structure shows how complementary the molecule is to the binding surface of the serine protease (Fig. 3C). 


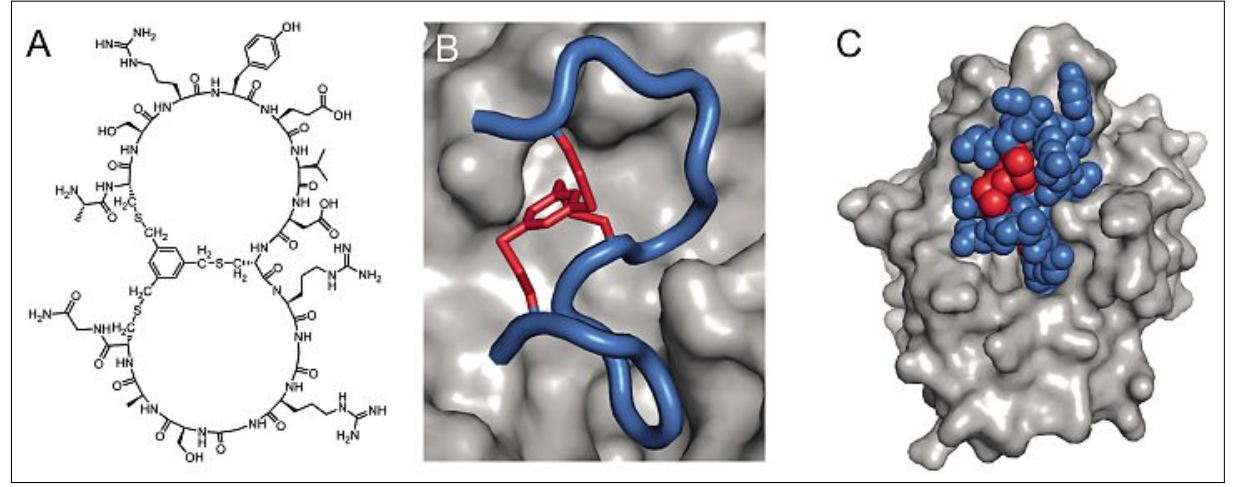

Fig. 3. Binding mode of a bicyclic peptide. (A) Structure of bicyclic peptide UK18 inhibiting the serine protease UPA with a $K_{\mathrm{i}}$ of $53 \mathrm{nM}$. (B and C) X-ray structure of UK18 co-crystalized with uPA (1.9 A resolution). The peptide moiety is shown in blue and the mesitylene linker in red. In panel $B$ the peptide backbone is represented as tube and the linker as stick model. In panel $\mathrm{C}$, the whole bicyclic peptide is shown as space fill model.

\section{Novel Bicyclic Peptide Formats}

Large libraries containing structurally diverse bicyclic peptides need to be screened to identify good binders to a wide range of targets. We chose to generate structurally highly diverse bicyclic peptide libraries by varying, in addition to the amino acid sequence, also the size of the peptide rings and the chemical linker that connects the cysteine residues in the peptides.

We first cloned phage peptide libraries with different combinations of ring sizes ranging from 3 to 6 random amino acids (Fig. 4A). Screening of these libraries yielded bicyclic peptides that bind to a large number of epitopes as evidenced by many different consensus sequences. ${ }^{[24]}$ While peptides isolated from the above described 6x6 library bound in average to one or two epitopes on a given target, bicyclic peptides pulled out of the new libraries bound to significantly more different epitopes. Some of the consensus sequences were found exclusively for specific ring size combinations.

Varying the chemical linker in the bicyclic peptides was a more complex task because peptide cyclization reagents needed to fulfill a number of criteria. Firstly, they must contain three reactive groups that efficiently and selectively react with thiols of cysteines. Secondly, the reaction must be compatible with a solvent mixture that is tolerated by filamentous phage as for example water or a mixture of water and an organic solvent. Thirdly, the reactive groups need to be arranged symmetrically to ensure formation of a single bicyclic peptide product for each peptide. We were able to develop two such new reagents based on bromoacetamide and acrylamide reactive groups and showed that they efficiently cyclize cysteine-rich peptides (Fig. 4B).[25] The novel linkers are now being applied in phage selections (unpublished results)
Recently, we showed that bicyclic peptides circularized through two disulfide bridges can be isolated by phage display. In phage selections with a $4 \times 4$ peptide library of the form $\mathrm{XCX}_{4} \mathrm{CX}_{4} \mathrm{CX}(\mathrm{X}=$ any amino acid, $\mathrm{C}=$ cysteine), we found that most of the isolated peptides contained a fourth cysteine in the randomized peptide region if the cyclization reagent TBMB was not added prior to the affinity selection. We hypothesized that the four cysteines form two pairs of disulfide bridges and thus bicyclic structures (Fig. 5A). Mass spectrometric analysis indicated that the four cysteines were indeed oxidized. As each peptide with four cysteines can form three disulfide bond isomers, we separated them chromatographically before characterization. X-ray structure analysis of such a peptide co-crystallized with the target protein confirmed the presence of two disulfide bridges and revealed the important structural role of the bicyclic configuration (collaboration with Prof. Petr Leiman, EPFL; Fig. 5B). An attractive feature of this bicyclic peptide phage selection approach is the large topological diversity of bicyclic peptides in the libraries. Peptides of the format $\mathrm{X}_{1} \mathrm{CX}_{\mathrm{m}} \mathrm{CX}_{\mathrm{n}} \mathrm{CX}_{\mathrm{o}}$ can form $3 x(1+m+n+o)$ different topologies as the $4^{\text {th }}$ cysteine can be placed in $1+m+n+o$ different positions and each peptide can form three disulfide bond isomers (Fig. 5C). Another nice feature is the technically simple procedure: the peptides are automatically bicyclized in the oxidative environment of the cell culture media during phage production.

\section{Entering the Sequence Space of Non-natural Amino Acids}

Non-natural amino acids can be incorporated into phage-displayed peptides ${ }^{[26]}$ but the procedure is technically complex. To date, phage peptide libraries with non-natural amino acids were not generated. However, small libraries of bicyclic peptides can be chemically synthesized and screened to generate ligands with improved binding affinity or metabolic stability. This is an important advantage of bicyclic peptides over antibodies that are not accessible to chemical synthesis. In a collaborative project with the group of Prof. Olivier Michielin (University of Lausanne) we used computational methods to predict beneficial amino acid substitutions in the

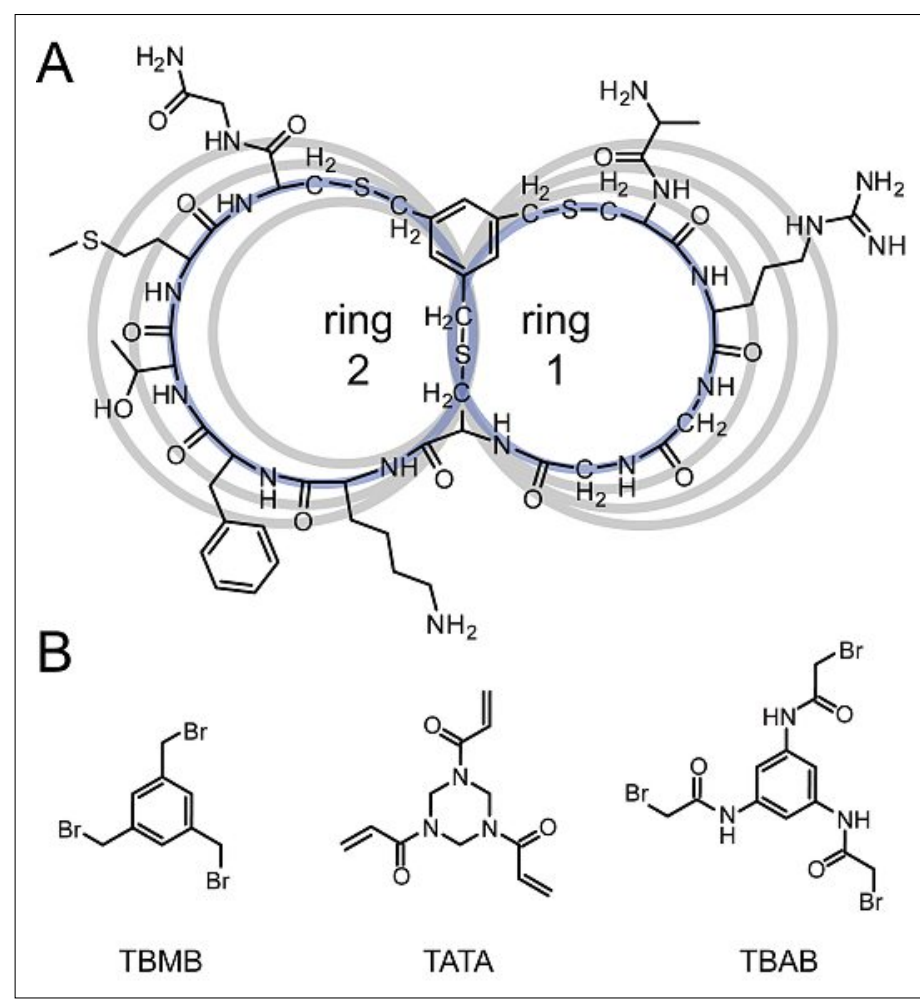

Fig. 4. Structural diversity of bicyclic peptide libraries. (A) Bicyclic peptide with 3 and 4 amino acids in the first and second ring, respectively. Bicyclic peptide phage libraries with a range of ring size combinations have been constructed. The size of the rings ranges between 3 and 6 amino acids as illustrated by the grey rings in the figure. (B) Three thiol-reactive chemical linkers suitable for the cyclization of cysteine-rich peptides. 


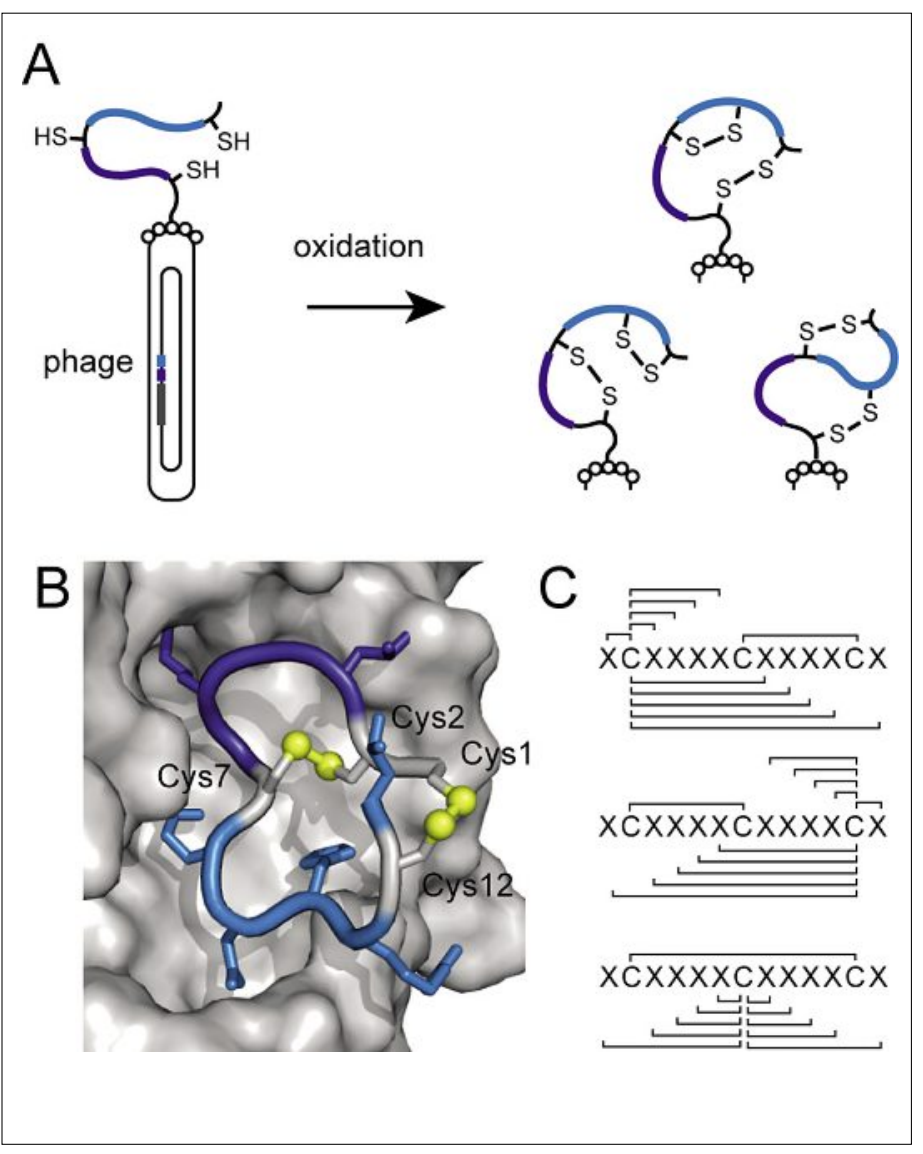

Fig. 5. Phage selection of bicyclic peptides based on two disulfide bridges. (A) Linear peptides with three cysteines in constant positions and two regions of random amino acids (in light and dark blue) are oxidized. Peptides containing a $4^{\text {th }}$ cysteine in one of the randomized regions can form two disulfide bridges and hence a bicyclic peptide structure.

(B) X-ray structure of the disulfide-cyclized peptide UK504 bound to UPA (1.5 Å resolution). (C) Illustration of the numerous peptide topologies that can be generated if peptides in a library of the form $\mathrm{XCX}_{4} \mathrm{CX}_{4} \mathrm{CX}$ contain a $4^{\text {th }}$ cysteine in the randomized region (X represents a random amino acid; $\mathrm{C}$ a cysteine residue). culation. In a range of experiments, we assessed the stability and various pharmacokinetic parameters of bicyclic peptides. Learning about these properties was important to know for which medical indications bicyclic peptides could potentially be applied. For example, antagonists blocking enzymes or receptors would need to circulate in the body for a longer time period and at a concentration well above the binding constant while an agonist may show good activity even if it stays in the body for only a short time.

Most bicyclic peptides incubated in blood plasma ex vivo were found to resist proteases for several hours and some even for several days. A study with the uPA inhibitor UK18 showed that a significant fraction of the bicyclic peptide remained completely intact while monocyclic and linear analogues were rapidly degraded. ${ }^{[28]}$ Interestingly, also the two rings of UK18 synthesized individually were less stable than the bicyclic peptide. This observation suggested that the two rings in a bicyclic peptide stabilize each other mutually, possibly by sterically hindering the access of proteases or by forming non-covalent interactions that reduce their conformational flexibility. ${ }^{[28]}$ bicyclic peptide UK18 that was crystallized with its target.[27] One of the two glycine residues in UK18 was found to have a positive phi dihedral angle in its target-bound form (Fig. 6A and 6B). As positive phi angles are favored by $\mathrm{D}$-amino acids, we replaced the glycine residue with a range of D-amino acids and found that D-serine enhances the inhibitory activity as well as the proteolytic stability. X-ray structure analysis of the mutant showed that it has the same backbone conformation as the parental bicyclic peptide (collaboration with Prof. Petr Leiman, EPFL; Fig. 6C and 6D). A retrospective analysis of cyclic peptides with known three-dimensional structure showed that several of them contain glycines with positive phi angles and suggested that substitution of glycine by Damino acids could be a general strategy to improve affinity and/or stability. While we have so far not exploited other non-natural amino acids, this would easily be possible as many Fmoc-amino acids are commercially available and the peptides are efficiently synthesized by automated parallel solid-phase peptide synthesis.

\section{Engineered Bicyclic Peptides with Long in vivo Half-life}

Peptide drug candidates often suffer from two major limitations, namely low proteolytic stability in bodily fluids and fast renal clearance from the blood cir-
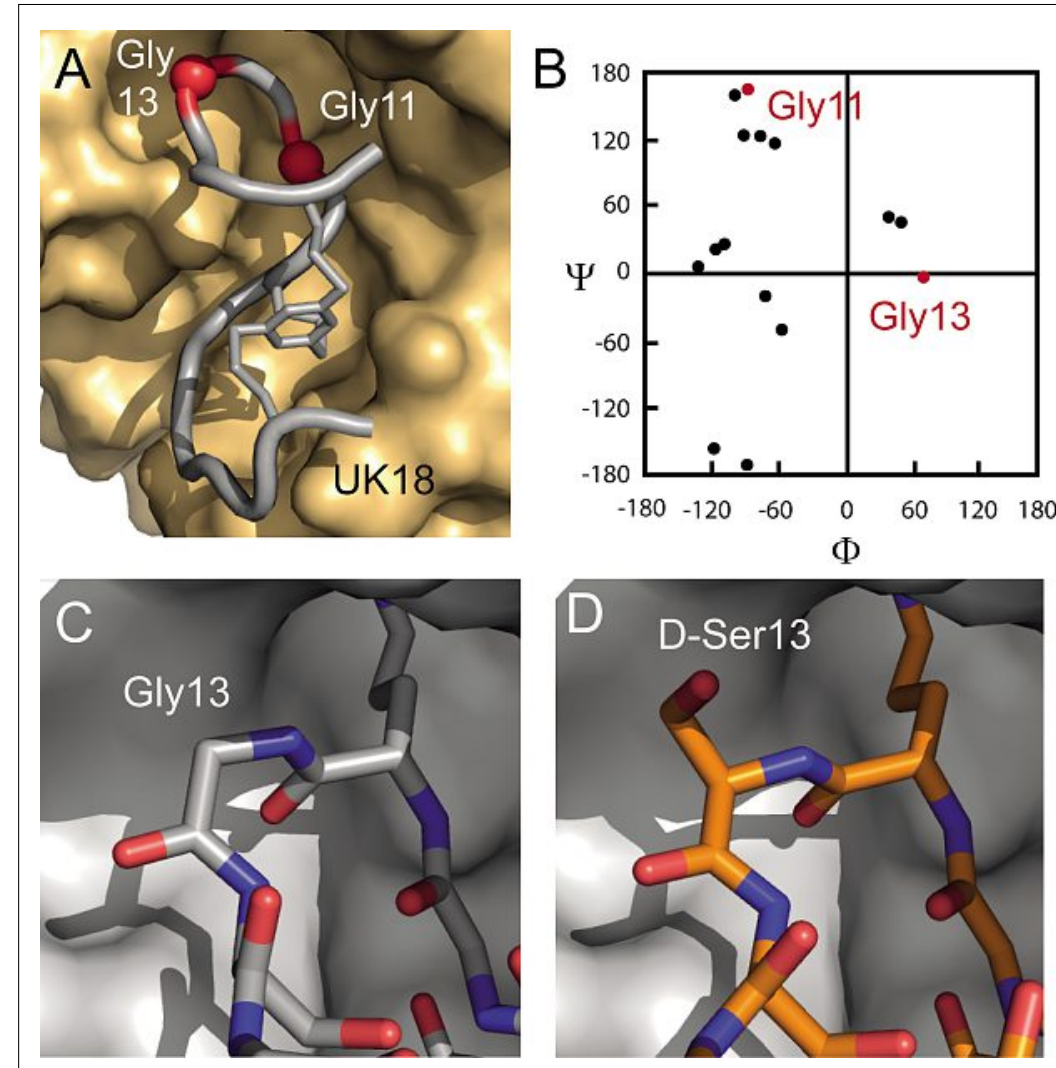

Fig. 6. Affinity maturation of a bicyclic peptide with non-natural amino acids. (A) The bicyclic peptide UK18 contains two glycine residues of which Gly13 has a positive phi dihedral angle when the peptide is bound to UPA. (B) Ramachandran plot showing phi and psi angles of UK18. The angles of the two glycine residues are highlighted. Gly13 has a phi and psi angle combination that is favored by D-amino acids. (C) X-ray structure of UK18 bound to UPA. The C-terminal peptide ring containing Gly13 is shown. (D) X-ray structure of UK202 bound to UPA. The C-terminal peptide ring containing $D-S e r 13$ is shown. Substitution of glycine to $D$-serine in position 13 did not change the backbone conformation of the bicyclic peptide but improved its affinity and stability. 
Bicyclic peptides applied intravenously to mice were found to be cleared by the kidney in relatively short time, as many approved peptide drugs. For example the elimination half-life of UK18 is 30 minutes (Fig. 7). Longer circulation times could be achieved by applying the bicyclic peptides subcutaneously. While the observed circulation times may be ideal for some agonists or for imaging applications, they would be too short for antagonists that need to inhibit targets for longer time periods. We thus applied two strategies to extend the circulation time of bicyclic peptides. In the first one, we conjugated the bicyclic peptide to an antibody Fc fragment that has a $\beta$ half-life of more than one day in mice and around one week in human. The bicyclic peptide-Fc fragment conjugate had an elimination half-life of 1.5 days, which was around 70 times longer than that of the 'naked' bicyclic peptide. ${ }^{[29]}$ The bicyclic peptide remained fully functional while circulating in the blood stream of mice for several days.

Conjugation of the bicyclic peptide to the Fc fragment increased its molecular mass from $2 \mathrm{kDa}$ to around $50 \mathrm{kDa}$ and offset advantages such as access to chemical synthesis and efficient tissue penetration. To generate bicyclic peptides with a smaller size and yet a long in vivo circulation time, we tested a less established strategy based on albumin-binding ligands. Albumin has an exceptionally long halflife of 19 days in human and around 2 days in mice. Non-covalent tethering of molecules to this highly abundant protein (the concentration in blood plasma is around $0.6 \mathrm{mM}$ in human) was found to extend their circulation time. ${ }^{[30]}$ We chemically conjugated the bicyclic peptide UK18 to a disulfide-cyclized albumin-binding peptide that binds mouse albumin with a $K_{\mathrm{d}}$ of around $30 \mathrm{nM} \cdot{ }^{[28,31]}$ The conjugate showed a much prolonged elimination half-life of 24 hours in mice. Mass spectrometric analysis revealed that the bicyclic peptide conjugate was not degraded at all, even after circulating in the blood stream of mice for several days. ${ }^{[28]}$ In a follow-up experiment, we found that non-covalent tethering of bicyclic peptides to albumin increases their stability. Despite exposure of the mice to high concentrations of bicyclic peptide ( $>10 \mu \mathrm{M}$ in blood) for extended time periods, we did not observe any toxic effects.

With both conjugation strategies, we could prolong the in vivo half-life of bicyclic peptides from 30 minutes to at least one day. Based on allometric scaling, this circulation time corresponds to a half-life of several days in humans. The substantial prolongation of the in vivo half-life was an important step in our work as this widened significantly the application range of bicyclic peptides.

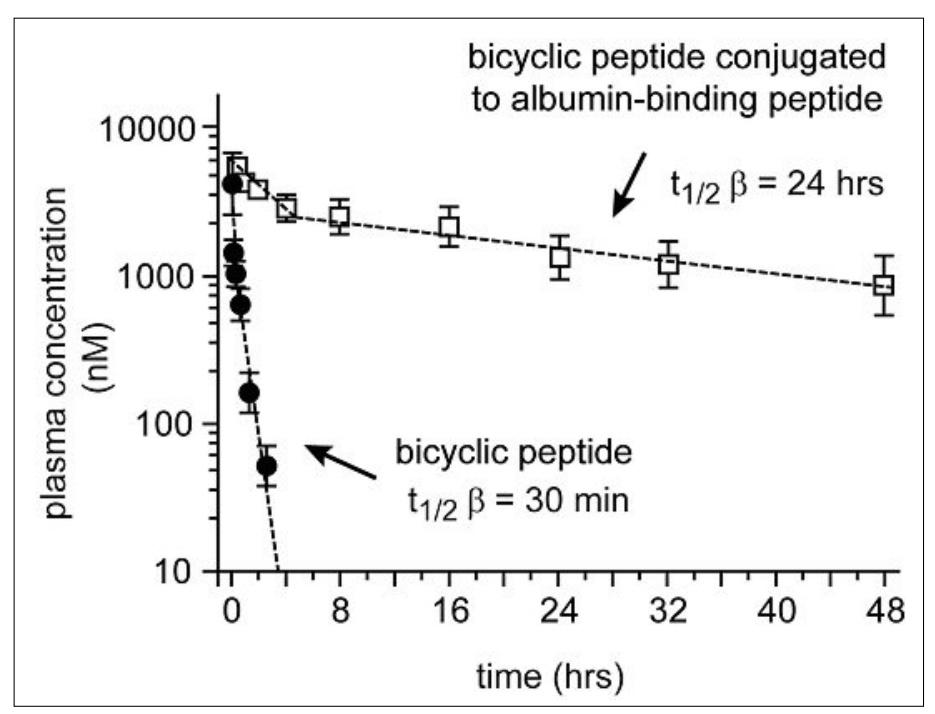

Fig. 7. Pharmacokinetic properties of bicyclic peptides. The bicyclic peptide UK18 has a $\beta$ half-life of 30 minutes in mice (i.v. administration). Although the peptide resists proteases in blood, it is rapidly cleared due to renal filtration. Conjugation of UK18 to an albumin-binding peptide (SA21) extended its $\beta$ half-life to $24 \mathrm{hrs}$. The bicyclic peptide conjugate is completely resistant to proteolytic activities in vivo.

A long term goal of our group is the development of orally available peptide drugs. While peptides can be formulated to pass the stomach efficiently, proteolytic degradation in the small intestine and inefficient transfer across the epithelium hinder their delivery into the blood stream. We reasoned that small amounts of peptide may be taken up in the small intestine if they were not degraded before. To increase the proteolytic stability, we incubated peptide-displaying phage particles with intestinal extracts of cow (pancreatin) prior to affinity selections. As target, we applied human plasma kallikrein, a serine protease playing a central role in hereditary angioedema. The selections yielded several bicyclic peptide inhibitors of plasma kallikrein with improved proteolytic stability. ${ }^{[32]}$

\section{Towards the Development of Therapeutics}

The ultimate goal of our laboratory is the development of therapeutics based on bicyclic peptides. Towards this end, we chose a number of diseases and the corresponding protein targets and isolated bicyclic peptide ligands from the above-described phage display libraries. An important criterion for the choice of medical indications was that peptide ligands offer advantages over competing molecule formats such as small molecules and monoclonal antibodies. While we initially developed bicyclic peptides to a range of proteases, we later showed that other types of proteins, as for example receptors or enzymes other than proteases, are also tractable. In the following, we report mostly published results about bicyclic protease inhibitors.

Two of our targets are the proteases uPA and matrix metalloproteinase 2 (MMP-2). Both of these proteases are over-expressed in several types of cancer and their activ- ity in tissue degradation and signaling facilitates tumor growth and/or invasion. The proteases have been difficult to target with small molecules and monoclonal antibodies for the following reasons. Small molecules inhibited paralogous proteases including several ones with physiologically important functions. Monoclonal antibodies did not efficiently diffuse into tissue due to their large size. In contrast, phageselected bicyclic peptides can differentiate between homologous proteases and due to their small size, they are expected to penetrate tumor tissue. The bicyclic peptide uPA inhibitor is described in the various sections above. ${ }^{[23]} \mathrm{We}$ are currently testing its ability to penetrate into tissues and block uPA over-expressed in human xenograft tumors in mice. The development of bicyclic peptide MMP-2 inhibitors turned out to be rather challenging. However, recently, we succeeded in developing a potent and highly selective MMP-2 inhibitor which we are currently applying in animal studies (unpublished data).

Other protease targets to which we have developed bicyclic peptide inhibitors are plasma kallikrein and human factor XIIa. Plasma kallikrein plays an important role in hereditary angioedema and diabetic retinopathy. The coagulation factor XIIa plays a role in thrombotic disorders. Both proteases, being expressed as zymogens, can activate each other and are thus physiologically and pathologically connected. Synthetic small molecular inhibitors with high selectivity for either of the two serine proteases are not available. In collaboration with Bicycle Therapeutics, a spin-out company based on the bicyclic peptide technology, our laboratory has generated bicyclic peptides that efficiently and selectively inhibit human and murine plasma kallikrein. ${ }^{[33]}$ The best inhibitor blocks human plasma kallikrein with a $\mathrm{Ki}$ of 300 pM. More recently, we developed also bicyclic peptide inhibitors of factor 
XIIa. The inhibitor selectively blocked the intrinsic coagulation pathway while not affecting extrinsic coagulation or interfering with other plasma proteases. ${ }^{[34]} \mathrm{We}$ are currently evaluating the inhibitors in vivo in collaboration with the group of Prof. Anne Angelillo-Scherrer (Insel Hospital Berne).

\section{Acknowledgements}

The financial contribution from the Swiss National Science Foundation (SNSF Professorship PP00P3_123524/1 to C.H.) is gratefully acknowledged. We also thank the National Competence Center for Biomolecular Imaging (NCCBI) for a $\mathrm{PhD}$ fellowship to P.D.

Received: October 10, 2013

[1] F. Giordanetto, J. Kihlberg, J. Med. Chem. 2013, DOI: $10.1021 / \mathrm{jm} 400887 \mathrm{j}$.

[2] http://cbc.arizona.edu/njardarson/group/toppharmaceuticals-poster.

[3] C. G. Ullman, L. Frigotto, R. N. Cooley, Brief. Funct. Genomics 2011, 10, 125.

[4] G. P. Smith, Science 1985, 228, 1315

[5] A. L. Nelson, E. Dhimolea, J. M. Reichert, Nat. Rev. Drug Discov. 2010, 9, 767.

[6] M. J. Rowley, K. O’Connor, L. Wijeyewickrema, Biotechnol. Annu. Rev. 2004, 10, 151.

[7] S. E. Cwirla, E. A. Peters, R. W. Barrett, W. J. Dower, Proc. Natl. Acad. Sci. USA 1990, 87, 6378.

[8] M. A. McLafferty, R. B. Kent, R. C. Ladner, W. Markland, Gene 1993, 128, 29.
[9] K. T. O’Neil, R. H. Hoess, S. A. Jackson, N. S. Ramachandran, S. A. Mousa, W. F. DeGrado, Proteins 1992, 14, 509.

[10] S. Chen, C. Heinis, 'Phage selection of mono- and bicyclic peptide ligands', in 'Biotherapeutics: Recent Developments using Chemical and Molecular Biology', RSC, 2013.

[11] H. B. Lowman, Annu. Rev. Biophys. Biomol. Struct. 1997, 26, 401.

[12] R. W. Roberts, J. W. Szostak, Proc. Natl. Acad. Sci. USA 1997, 94, 12297.

[13] K. Josephson, M. C. Hartman, J. W. Szostak, J. Am. Chem. Soc. 2005, 127, 11727.

[14] H. Murakami, A. Ohta, H. Ashigai, H. Suga, Nat. Methods 2006, 3, 357.

15] Y. Yamagishi, I. Shoji, S. Miyagawa, T. Kawakami, T. Katoh, Y. Goto, H. Suga, Chem. Biol. 2011, 18, 1562.

[16] F. T. Hofmann, J. W. Szostak, F. P. Seebeck, $J$ Am. Chem. Soc. 2012, 134, 8038

[17] Y. V. Schlippe, M. C. Hartman, K. Josephson, J. W. Szostak, J. Am. Chem. Soc. 2012, 134 10469.

[18] T. Kawakami, T. Ishizawa, T. Fujino, P. C. Reid, H. Suga, H. Murakami, ACS Chem. Biol. 2013, in print.

[19] S. Fishbane, B. Schiller, F. Locatelli F, A. C. Covic, R. Provenzano, A. Wiecek, N. W. Levin, M. Kaplan, I. C. Macdougall, C. Francisco, M. R. Mayo, K. R. Polu, A.-M. Duliege, A. Besarab, New Engl. J. Med. 2013, 368, 307.

[20] I. C. Macdougall, R. Provenzano, A. Sharma, B. S. Spinowitz, R. J. Schmidt, P. E. Pergola, R. I. Zabaneh, S. Tong-Starksen, M. R. Mayo, H Tang, K. R. Polu, A.-M. Duliege, S. Fishbane, New Engl. J. Med. 2013, 368, 320.
[21] C. Heinis, T. Rutherford, S. Freund, G. Winter, Nat. Chem. Biol. 2009, 5, 502.

[22] I. Rentero Rebollo, C. Heinis, Methods 2013, 60,46 .

[23] A. Angelini, L. Cendron, S. Chen, J. Touati, G. Winter, G. Zanotti, C. Heinis, ACS Chem. Biol. 2012, 7, 817 .

[24] I. R. Rebollo, A. Angelini, C. Heinis, MedChem Comm 2013, 4, 145.

[25] S. Chen, J. Morales-Sanfrutos, A. Angelini, B. Cutting, C. Heinis, ChemBiochem 2012, 13, 1032.

[26] F. Tian, M. L. Tsao, P. G. Schultz, J. Am. Chem. Soc. 2004, 126, 15962.

[27] S. Chen, D. Gfeller, S. A. Buth, O. Michielin, P. G. Leiman, C. Heinis, ChemBiochem 2013, 14, 1316.

[28] A. Angelini, J. Morales-Sanfrutos, P. Diderich, S. Chen, C. Heinis, J. Med. Chem. 2012, 55, 10187.

[29] A. Angelini, P. Diderich, J. Morales-Sanfrutos, S. Thurnheer, D. Hacker, L. Menin, C. Heinis, Bioconjugate Chem. 2012, 23, 1856.

[30] L. Pollaro, C. Heinis, MedChem Comm 2010, 1, 319.

[31] M. S. Dennis, M. Zhang, Y. G. Meng, M. Kadkhodayan, D. Kirchhofer, D. Combs, L. A. Damico, J. Biol. Chem. 2002, 277, 35035.

[32] V. Baeriswyl, C. Heinis, Protein Eng. Des. Sel. 2013, 26, 81 .

[33] V. Baeriswyl, H. Rapley, L. Pollaro C. Staoe, D. Teufel, E. Walker, S. Chen, G. Winter, J. Tit, C. Heinis ChemMedChem 2012, 7, 1173.

[34] V. Baeriswyl, S. Calzavarini, C. Gerschheimer, P. Diderich, A. Angelillo-Scherrer, C. Heinis, J. Med. Chem. 2013, 56, 3742. 